\title{
Farmacoeconomia: uma ferramenta para a gestão dos gastos com medicamentos em hospitais públicos
}

\author{
Pharmacoeconomics: a tool for the management \\ of drugs expenditures in public hospitals
}

Recebido em: 21/01/2014 Aceito em: 25/09/2014

Priscila Becker PACKEISER, Darielli Gindri RESTA Universidade Federal de Santa Maria. Avenida Roraima, 1000 Camobi. Santa Maria, RS, Brasil. CEP 97105-900 E-mail:priscilabecker@gracas.org.br

\begin{abstract}
Pharmacoeconomics is the application of economics to the study of pharmaceuticals optimizing the financial expenditure without impairment to the treatment of the patient. Different pharmaeconomics studies can be employed, such as: cost minimization, cost-utility analysis, cost-benefit and cost-effectiveness. The objective of this study was to review the concepts of pharmacoeconomics and pharmacoeconomics, showing the main characteristics and examples of applicability in hospitals. A literature survey was conducted in the databases Scielo, Lilacs and Medline, selecting 16 articles in the period of January to March 2014, published in English, Portuguese and Spanish. The scarcity and limited resources of Brazilian public hospitals coupled with the aging population make pharmacoeconomics an important tool for decision making regarding the pharmacological features, letting you choose the best treatment option for patient, with the best cost-benefit to the hospital.
\end{abstract}

Keywords: pharmacoeconomics; pharmaeconomic analysis; hospital

\section{RESUMO}

Farmacoeconomia é a aplicação da economia ao estudo dos medicamentos, otimizando os gastos financeiros sem prejuízo ao tratamento do paciente. Diferentes estudos farmacoeconômicos podem ser empregados, sendo que os principais são: minimização de custo, análise custo-utilidade, custo-benefício e custo-efetividade. O objetivo deste estudo foi realizar uma revisão dos conceitos de farmacoeconomia e dos estudos farmacoeconômicos, apresentando suas principais características e exemplos de aplicabilidade no âmbito hospitalar. Foi realizado um levantamento bibliográfico nas bases de dados do Scielo, Lilacs e Medline, selecionando 16 artigos no período de janeiro a março de 2014, publicados em inglês, português e espanhol. A escassez e limitação de recursos dos hospitais públicos brasileiros aliado ao envelhecimento populacional torna a farmacoeconomia uma importante ferramenta para a tomada de decisão quanto aos recursos farmacológicos, permitindo a escolha da melhor opção de tratamento ao paciente, adequada à possibilidade de custeio do hospital.

Palavras-Chave: farmacoeconomia; análise farmacoeconômica; hospital 


\section{INTRODUÇÃO}

Os medicamentos consomem uma parcela considerável de recursos de um país, tendo um forte impacto sobre as despesas totais de saúde (1). O aumento da expectativa de vida, aliado a fatores como o uso concomitante de múltiplos fármacos e a falta de conhecimento e orientação da população, os gastos com saúde vêm crescendo em ritmo acelerado em âmbito mundial, preocupando usuários, governos e sociedade (2).

Apesar de a indústria farmacêutica ser responsável por $8 \%$ do Produto Interno Bruto (PIB) do Brasil, tendo grande importância para a economia, a elevação da despesa pública com medicamentos tem sido motivo de preocupação entre os governos porque, em geral, estes aumentos são superiores à inflação ou ao PIB (1). De acordo com os últimos dados estatísticos publicados, a estimativa de gasto em saúde nos últimos anos foi de $8,4 \%$ do PIB, sendo $41,6 \%$ destes gastos realizados pelo sistema público (3).

No âmbito do Sistema Único de Saúde (SUS), com o envelhecimento populacional e as transformações nas estruturas de morbimortalidade da população, ocorreu uma elevação das doenças crônico-degenerativas e suas sequelas, havendo maior demanda por serviços de saúde e, consequentemente, aumento dos gastos com a mesma $(2,4)$. Esse aumento contínuo de custos resultou em uma necessidade de compreender como recursos limitados podem ser utilizados de maneira mais eficiente e efetiva (5). Assim, a aplicabilidade social dos estudos farmacoeconômicos tornou-se uma estratégia importante face à dificuldade de custear atendimento completo do paciente por livre escolha de terapêutica dentro do SUS (6).

A análise farmacoeconômica se apresenta como uma ferramenta capaz de fornecer informações para ajudar os gestores a comparar alternativas e decidir sobre a melhor opção para as necessidades do serviço de saúde, associando racionalização de despesas e eficiência clínica (1). Além da mensuração dos custos, a farmacoeconomia também analisa os benefícios, que podem ser econômicos ou não econômicos, tais como efeitos na saúde, aumento na expectativa e na qualidade de vida (6).

O objetivo deste artigo é realizar uma revisão dos principais conceitos utilizados na farmacoeconomia, elencar os tipos de estudos farmacoeconômicos existentes, suas características, aplicabilidades e limitações, assim como investigar avaliações farmacoeconômicas realizadas em outros estudos, apresentando idéias que possam ser aplicadas para a melhoria da gestão dos gastos com medicamentos nos hospitais públicos.

\section{MÉTODO:}

Foi realizada uma revisão da literatura por meio de levantamento bibliográfico no período de janeiro a março de 2014, buscando publicações indexadas nas bases de dados do Scielo, Lilacs e Medline. Foram escolhidas essas bases de dados por serem as principais fontes de publicações na área da saúde.

Foram adotados os seguintes critérios de inclusão para a seleção de artigos: artigos com texto completo disponível para acesso; todas as categorias de artigo (original, revisão de literatura, reflexão, atualização, relato de experiência, etc.); artigos publicados em português, inglês ou espanhol; no período compreendido entre os anos de 2000 a 2014; que contivessem em seu título e/ou resumo os descritores "farmacoeconomia" ou "análise farmacoeconômica" e "hospital" para os artigos em português, "pharmacoeconomy" ou "pharmacoeconomics studies" e "hospital" para os artigos em inglês e "farmacoeconomía" ou "estúdios farmacoeconómicos" e "hospital" para as publicações em espanhol; e tivessem como assunto principal os temas farmacoeconomia, serviço de farmácia hospitalar e custos de cuidados em saúde. O descritor "hospital" foi utilizado na busca considerando que o objetivo é analisar como se pode utilizar a farmacoeconomia no âmbito hospitalar para a otimização dos gastos.

Foram excluídos da pesquisa os artigos que não atendiam aos critérios de inclusão anteriormente mencionados, aqueles não relacionados com o objetivo-tema desta revisão bibliográfica, os publicados em outro formato que não artigo científico (tese, dissertação, carta, resenha, etc.), publicados em período anterior ao ano de 2000 , em outro idioma senão aos relacionados na metodologia, bem como também os artigos que não estavam disponíveis na íntegra para leitura. A partir da leitura dos resumos, foram excluídos ainda os artigos que não tinham relação com o objetivo deste estudo, que não possuíam informações relevantes que complementassem o levantamento bibliográfico ou que não tivessem aplicabilidade na área hospitalar. Alguns artigos com estudos farmacoeconômicos semelhantes também foram excluídos devido à repetitividade de informações. Após a seleção, foi feita uma leitura minuciosa dos artigos, para verificar a adequação com o tema da pesquisa e excluir aqueles que ainda não estivesse de acordo com os critérios de inclusão estabelecidos.

Na base de dados Scielo, foram identificados 14 artigos, dos quais cinco se encaixavam nos critérios de inclusão; no Lilacs, dos 31 artigos, foram selecionados três; e no Medline, dos 61 artigos encontrados, apenas 
oito foram selecionados, resultando em uma amostra final de 16 artigos. Cabe salientar que a busca foi realizada na ordem apresentada, sendo que as publicações indexadas em mais de uma base de dados, foram selecionadas apenas na primeira busca.

Após a seleção dos artigos, foi realizada uma busca em uma biblioteca da universidade local selecionando-se as bibliografias cujo título fosse "farmacoeconomia" ou "economia em saúde". A partir disso, foi selecionado também um livro-texto recente e um material publicado pelo Ministério da Saúde, considerando seu conteúdo e informações relevantes para a pesquisa.

\section{RESULTADOS E DISCUSSÃO}

Farmacoeconomia. A farmacoeconomia é a aplicação da economia ao estudo dos medicamentos com a otimização da utilização de recursos financeiros sem prejuízo à qualidade do tratamento. Em outras palavras, é a descrição, a análise e a comparação dos custos e das consequências de terapias medicamentosas para os pacientes, os sistemas de saúde e a sociedade, com o objetivo de conciliar as necessidades terapêuticas com as possibilidades de custeio $(4,7)$.

É uma ferramenta que ajuda a selecionar as opções mais eficientes e que auxilia na distribuição de recursos para a saúde de uma forma mais justa e equilibrada. Além disso, contribui para o uso racional de medicamentos por meio da incorporação do custo a questões sobre segurança, eficácia e qualidade dos diferentes tratamentos médicos, buscando uma melhor relação entre custos e resultados (1).

A tarefa primordial da economia em saúde não é, ao contrário do que acreditam alguns, reduzir os custos em saúde "custe o que custar", mas, sobretudo, utilizando os recursos escassos, desenvolver ações que sejam capazes de propiciar os melhores resultados para a saúde da população, ao menor custo possível (8).

O primeiro elemento de um estudo de farmacoeconomia é o custo, sempre um dado complexo, que engloba elementos mensuráveis, tanto qualitativa quanto quantitativamente, mas que também nem sempre apresenta clara tangibilidade, como em aspectos relacionados à qualidade de vida (7). Conforme Tonon e cols. (2008), o custo representa o valor de todos os insumos (trabalho, materiais, dispositivos, medicamentos, entre outros) utilizados na produção ou distribuição de bens ou serviços e envolve todos os recursos relevantes na aplicação do tratamento. São classificados em custos diretos, indiretos e intangíveis (9).
Os custos diretos são aqueles diretamente relacionados aos serviços de saúde que implicam em dispêndios imediatos, sendo de identificação imediata $(2,9,10)$. Contemplam produtos e serviços desenvolvidos para prevenir, detectar e/ou tratar uma doença, entre eles, os medicamentos, envolvendo todo gasto relacionado com a aquisição, transporte, administração e descarte destes $(2,11)$. Como exemplo desse tipo de custo, um antibiótico, que é administrado por via endovenosa, onde os custos diretos são referentes à sua compra, ao armazenamento em locais adequados, aos custos dos materiais para administrá-lo, ao trabalho dos profissionais para preparar e administrar o medicamento, etc. Assim, antibióticos cuja posologia requeira várias administrações durante o dia possuem custos diretos aumentados em relação a outros que embora tenham maior preço, possuem menor frequência diária de administração (11).

Os custos indiretos são relativos às mudanças da capacidade produtiva do indivíduo e familiares decorrentes do processo de adoecimento ou de mortalidade precoce. Representam, por exemplo, os custos dos dias de trabalho perdido, incapacidade de realizar as atividades profissionais, tempo gasto em viagens para receber cuidado médico e morte prematura decorrente da doença $(2,9,10)$. Estão relacionados também com as reações adversas ou à condições de administração de um medicamento. Como exemplo, determinados medicamentos que são administrados por via intravenosa e que obrigatoriamente condicionam que o paciente seja internado, apresentam custo maior em relação aos medicamentos intramusculares que podem ser administrados em ambulatório sem afetar a produtividade do paciente(11).

Os custos intangíveis são aqueles de difícil mensuração monetária. Embora muito importantes para os pacientes, necessitam ainda de significado econômico. São os custos do sofrimento, da dor, da tristeza, ansiedade, fadiga ou a redução da qualidade de vida, devidos a uma doença ou tratamento de uma doença $(2,5,9,10)$. A diferença dos custos se mede de diferentes maneiras de acordo com o estudo empregado. O que se busca quando há distintas alternativas de tratamento é a obtenção dos maiores efeitos positivos possíveis ao menor custo possível(11).

Para um entendimento completo das técnicas de avaliação farmacoeconômica, é importante descrever também os conceitos de eficácia, efetividade e eficiência, que frequentemente são confundidos em sua terminologia ou definição $(1,7)$. A eficácia refere-se aos benefícios, consequências e resultados de medicamentos utilizados sob condições ideais, ou seja, durante a aplicação em ensaios clínicos, utilizando critérios rigorosos de seleção de pacientes, e o acompanhamento da 
evolução clínica em estrita conformidade com o regime terapêutico (1).

A efetividade mede os resultados da utilização de medicamentos na prática clínica diária, avaliando-o, assim, em condições de rotinas, nos quais múltiplas interferências podem modificar a resposta. A efetividade é, frequentemente, menor que a eficácia $(1,7)$. Assim, a efetividade de um medicamento dependerá de fatores associados às condições da prática clínica diária como, por exemplo, a probabilidade de resistência cruzada entre antibióticos, de erros na administração dos medicamentos e de falha na adesão ao tratamento pelo paciente (10).

A eficiência é a confirmação da efetividade sob custos razoáveis; é o sinônimo de custo-efetividade. Os estudos farmacoeconômicos, realizados com dados de eficácia, podem apresentar diferenças quando as influências externas afetam os resultados clínicos, sendo mais adequadamente realizados após a adequada comprovação de efetividade $(1,7)$. É a obtenção máxima de be- nefício com o recurso financeiro empregado e para que seja obtida a eficiência econômica, é necessário atingir a efetividade clínica máxima, ou seja, melhorar os desfechos sem aumentar a quantia de recursos investidos (9).

Avaliação farmacoeconômica. Nos últimos anos, os governos de vários países vêm adotando algumas estratégias no campo da saúde em resposta, principalmente, ao crescente aumento dos gastos farmacêuticos nos seus sistemas sanitários. Entre as medidas orientadas a reduzir os gastos em farmácia, destacam-se o fortalecimento da atenção farmacêutica e a realização de estudos de avaliação econômica aplicados aos medicamentos (10).

São quatro os tipos de análises recomendados pela farmacoeconomia: minimização de custos, custo-benefício, custo-efetividade e custo-utilidade (2). O Quadro 1 apresenta os tipos de análises farmacoeconômicas e as unidades de medida de custo e de desfecho de cada análise.

Quadro 1. Tipos de análises farmacoeconômicas (5)

\begin{tabular}{|c|c|c|}
\hline Metodologia & Unidade de medição de custo & Unidade de medição de desfecho \\
\hline Análise de minimização de custos & Unidades monetárias & Pressuposta como equivalente em grupos comparáveis \\
\hline Análise de custo-efetividade & Unidades monetárias & $\begin{array}{l}\text { Unidades naturais (anos de vida ganhos, pressão } \\
\text { sangüínea }[\mathrm{mmHg}] \text {, glicose no sangue }[\mathrm{mMol} / \mathrm{L}] \text { ) }\end{array}$ \\
\hline Análise de custo-benefício & Unidades monetárias & Unidades monetárias \\
\hline Análise de custo-utilidade & Unidades monetárias & $\begin{array}{l}\text { Anos de vida ajustados pela qualidade ou outras } \\
\text { utilidades }\end{array}$ \\
\hline
\end{tabular}

adaptado de Rascati (2010)

Minimização de custo. É a forma mais simples de avaliação econômica - somente os custos são submetidos à comparações, pois a eficácia ou efetividade das alternativas comparáveis são iguais (2).

O primeiro critério antes da realização da análise de minimização de custo é determinar a equivalência terapêutica das alternativas. Caso sejam diferentes, não é possível a realização deste tipo de estudo(1). Os tipos de intervenções que podem ser avaliados com esse método são limitados (5). É o tipo de análise útil na comparação de doses e vias de administração diferentes, considerando que o resultado de duas ou mais alternativas são as mesmas em relação aos resultados clínicos $(1,4)$.

Um outro exemplo inclui a medição dos custos de recebimento de um medicamento em diferentes ambientes. Por exemplo, os custos de recebimento de um antibiótico intravenoso pode ser medido e posteriormente, comparado aos custos de recebimento de mesmo antibiótico (nas mesmas doses) em casa, por um serviço de assistência domiciliar à saúde (5).

Tabela 1. Análise de minimização de custo das bandagens A e B (9)

\begin{tabular}{|l|c|c|}
\hline \multicolumn{1}{|c|}{ Tipo de custo } & Bandagem $\mathbf{A}$ & Bandagem B \\
\hline Preço da embalagem & $\$ 30$ & $\$ 58$ \\
\hline Número de bandagens por embalagem & 20 & 24 \\
\hline Preço unitário & $\$ 1,50$ & $\$ 2,41$ \\
\hline Troca diária (número de bandagens/dia) & 4 & 3 \\
\hline Custo diário & $\$ 6$ & $\$ 7,23$ \\
\hline Tempo de tratamento & 7 dias & 7 dias \\
\hline Custo do tratamento fracionado & $\$ 42$ & $\$ 50,61$ \\
\hline Custo do tratamento (embalagem fechada) & $\$ 60$ & $\$ 58$ \\
\hline
\end{tabular}


Na Tabela 1, é apresentada a comparação do custo de dois tipos de bandagens (A e B) utilizadas na terapia tópica de feridas. As bandagens são tecnicamente idênticas, proporcionando as mesmas condições e tempo de cicatrização quando comparadas em ensaios clínicos. São vendidas em embalagens fechadas, com preços e quantidades distintas por embalagens. Nesse exemplo, para comparar o custo da terapia, é necessário considerar o número de bandagens utilizadas por dia, a duração média da terapia, e a aquisição desse material (9).

Analisando o quadro no âmbito hospitalar, pode ser verificado que o preço unitário e o custo diário da terapia são mais caros para a bandagem $\mathrm{B}$; apesar do custo do tratamento com a embalagem A fechada ser maior, o custo com a embalagem fracionada é menor, tornando essa opção mais vantajosa, pois vários pacientes serão submetidos à mesma terapia tópica (9).
Outro exemplo de análise de minimização de custo, é o estudo desenvolvido por Rascati (2010), no qual foi analisado o custo da administração de carboplatina, um agente quimioterápico, em doses fracionadas, e da administração de carboplatina com um antiemético em dose única (5). Devido a problemas com a náusea induzida pela quimioterapia, é recomendado que seja administrada a metade da dose mensal de carboplatina necessária para cada ciclo, com um intervalo de cinco dias entre cada uma. Em estudos complementares, foi observado que, se fosse dado aos pacientes um medicamento anti-emético, poderia ser administrada a dose total de carboplatina em uma só consulta, e a efetividade clínica do tratamento quimioterápico foi idêntica nos dois métodos de administração.

No estudo, foram calculados os custos de prescrição, das infusões intravenosas e dos atendimentos médicos ou ambulatoriais, conforme a Tabela 2.

Tabela 2. Custos do primeiro ciclo de tratamento com carboplatina.

\begin{tabular}{|c|c|c|}
\hline & $\begin{array}{l}\text { Dose fracionada de } \\
\text { carboplatina }\end{array}$ & $\begin{array}{l}\text { Dose plena de carboplatina } \\
+ \text { antiemético }\end{array}$ \\
\hline Custo médio da carboplatina & $\$ 2964$ & $\$ 2980$ \\
\hline Custo médio do antiemético & $\mathrm{N} / \mathrm{A}$ & $\$ 40$ \\
\hline Custo da administração intravenosa & $\$ 160$ & $\$ 80$ \\
\hline Custo do atendimento por médico ou atendimento ambulatorial & $\$ 128$ & $\$ 64$ \\
\hline Custo total por paciente & $\$ 3252$ & $\$ 3164$ \\
\hline
\end{tabular}

Apesar dos custos dos medicamentos serem mais altos no grupo com o anti-emético, esse aumento foi neutralizado pela diminuição dos custos de administração e atendimento por médicos. A economia possibilitada pela administração de uma dose por ciclo foi de aproximadamente $\$ 88$. Além disso, apesar de somente os custos diretos médicos terem sido avaliados, se fossem incluídas a economia do paciente (menores custos de deslocamento) e da sociedade (é possível maior produtividade do paciente se menor tempo for gasto no consultório médico ou na clínica), a vantagem econômica da opção de administração de uma dose por ciclo seria ainda maior(5).

Custo-benefício. Expressa custos e resultados reais e potenciais (individual ou coletivo) da implementação de um programa, exclusivamente em unidades monetárias $(2,4)$. Dentre as análises, é a que possui a mais longa história no contexto das avaliações econômicas, pois foi amplamente utilizada no setor público como estratégia de avaliação de viabilidade econômica de projetos sociais, quando comparados em unidades monetárias (2).

Nas análises de custo-benefício é atribuído aos benefícios ou impactos de uma ação em saúde um valor monetário. Os resultados dessa análise são apresentados em benefí- cios líquidos (benefícios da intervenção menos os custos da intervenção). Os benefícios líquidos podem ser utilizados e comparados com uma variedade de atividades não similares de impactos para a saúde, uma vez que esses impactos também seriam mensurados em valor monetário(12).

Uma vantagem desse tipo de análise é que muitos desfechos diferentes podem ser comparados desde que as medidas de desfechos sejam valoradas em unidades monetárias (5).

Os resultados desse tipo de estudo indicam se uma estratégia específica direciona para o ganho líquido ou perda líquida (12). Entretanto, é cada vez menos utilizado devido à dificuldade de transformar dimensões intangíveis como, por exemplo, sofrimento ou morte, em unidades monetárias (2), como pela limitação de transformar monetariamente um benefício clínico, como salvar uma vida e reduzir a probabilidade de morte e atribuir valores a vida de uma pessoa idosa e a vida de uma criança, ou uma vida com limitações físicas e uma sem incapacidade (12).

Zahdi e cols. (2009), avaliaram a relação custo-benefício da vacinação contra hepatite A no Estado do 
Paraná de 2000 a 2003. Foram 14682 casos notificados, sendo que $82,4 \%$ dos casos e $50 \%$ dos óbitos ocorreram em menores de 15 anos. Foi feito um levantamento de todos os gastos decorrentes do tratamento, suas complicações, internações hospitalares e exames laboratoriais. O custo total para o tratamento de pacientes com hepatite A foi de US\$8.076.408,00 (13).

Com base na análise do custo da vacina e na necessidade de duas doses para a obtenção da imunidade por longo tempo, foi calculado um gasto de US\$3.552.700,00. Considerando uma incidência de 3\% de eventos adversos à vacina, que requereriam monitoramento por profissional de saúde, o custo total seria de US\$3.569.752,00.

Comparando os valores estimados em gastos com a doença e o custo da vacinação, foi observado que para cada dólar investido na vacina, eram economizados 2,26 dólares com a doença; $\mathrm{O}$ estudo mostrou que a vacinação contra hepatite A era definitivamente vantajosa na profilaxia contra a infecção viral, além de prevenir danos sociais, evitar mortes e prevenir epidemias, existindo relação custo-benefício com a implantação da imunização (13).

Custo-efetividade. $\mathrm{Na}$ análise de custo-efetividade, os custos são medidos em unidades monetárias e os desfechos em unidades naturais de saúde que indicam melhorias de saúde (5). As unidades de medida utilizadas para mensurar os benefícios terapêuticos podem ser: anos de vida ganhos, casos detectados, dias de incapacidade evitados, unidades de pressão arterial reduzidas, número de doenças evitadas, etc. A unidade de medida selecionada deve ser aquela com o impacto mais relevante para a análise $(10,12)$.

O objetivo da análise custo-efetividade é avaliar o impacto de distintas alternativas de intervenção à saúde, permitindo melhorar os efeitos do tratamento em troca da aplicação de recursos adicionais $(2,17)$. Portanto, ela é sempre comparativa e se destina à escolha da melhor estratégia para se atingir um mesmo objetivo, quer seja ele de prevenção, diagnóstico ou tratamento (12). É o tipo de análise mais utilizada atualmente na farmacoeconomia, porque possibilita o uso na prática cotidiana das mesmas unidades utilizadas nos ensaios clínicos (2).

Este estudo tem como maior limitação seu aspecto pontual, ou seja, de considerar apenas o objetivo final do estudo, como a mortalidade, e não sua repercussão sobre a qualidade de vida dos pacientes (re-internações, limitações físicas, etc.) que pode ser melhor ou pior em relação a sua efetividade (7).

A medida dos benefícios terapêuticos em uma análise custo-efetividade dependerá do objetivo da farmacoterapia. Assim, se o estudo tratar da avaliação de um novo medicamento para a hipercolesterolemia utilizará como indicador clínico, por exemplo, a redução da taxa de colesterol em mg/dL. No caso de comparar três alternativas de tratamento farmacológico da hipertensão arterial (um diurético, um beta-bloqueador e um inibidor da enzima conversora da angiotensina), as medidas comuns de efetividade poderão ser: redução da pressão arterial em $\mathrm{mmHg}$ (indicador intermediário) ou número de eventos cardiovasculares evitados (indicador final). Dessa forma, os indicadores são parâmetros utilizados para evitar as mudanças ocorridas no estado de saúde (efeitos) de um indivíduo ou grupo de pessoas frente a uma tecnologia sanitária (10).

A principal desvantagem desse tipo de análise é que os desfechos das alternativas utilizadas na comparação devem ser medidas nas mesmas unidades clínicas. Não pode ser utilizada a análise de custo-efetividade para comparar os desfechos de um produto que combata a hipertensão (que poderá medir mudanças em $\mathrm{mmHg}$ para determinar o desfecho) com os de um produto para tratamento da asma (que poderá medir o volume expiratório forçado para a determinação do desfecho). Além disso, mesmo que produtos para doenças ou condições semelhantes sejam comparados, mais de um tipo de desfecho clínico poderá ser importante. Por exemplo, ao se medir os efeitos de terapias que utilizam reposição hormonal, o efeito sobre os sintomas da menopausa e sobre as medidas de densidade mineral óssea poderão ficar em evidência, justificando o cálculo de diversas razões custo-efetividade para a comparação (5). Do ponto de vista de tomada de decisão, a análise com unidades diferentes pode ser difícil e especialmente arbitrária, em que a valorização ocorre de modo subjetivo pelo gestor e não explícito pelo usuário (12).

No exemplo mostrado na Tabela 3, há os resultados de uma análise de custo-efetividade, onde apresenta a comparação entre esquemas analgésicos utilizados por pacientes no pós-operatório. As opções terapêuticas incluíam esquema unimodal (um analgésico) e multimodal (dois analgésicos). Neste estudo, para o cálculo dos custos diretos, foram considerados os analgésicos, os materiais utilizados no preparo e administração dos medicamentos, o cateter venoso periférico (quando houve via intravenosa) e o tempo de enfermagem. Para cálculo da efetividade foi utilizado o consumo de analgésico opióide no regime suplementar (9). A análise custo-efetividade foi expressa pelo custo por paciente que não utilizou analgesia suplementar nas 24 horas. Por meio da Tabela 3, é possível concluir que o esquema mais caro foi o esquema A e o mais barato foi o C. No entanto, o de melhor relação custo-efetividade médio foi o $\mathrm{E}(\$ 39,14)$, seguido do A $(\$ 45,74)$. 
Tabela 3. Análise de custo-efetividade de esquemas analgésicos

\begin{tabular}{|c|c|c|c|}
\hline Esquema analgésico & Custo $\mathbf{( \$ )}$ & Efetividade & Custo-efetividade \\
\hline A & 2722,00 & 59,50 & 45,74 \\
\hline B & 2671,00 & 42,90 & 62,26 \\
\hline C & 1544,00 & 23,10 & 66,84 \\
\hline D & 2673,00 & 40,00 & 66,82 \\
\hline E & 1957,00 & 50,00 & 39,14 \\
\hline
\end{tabular}

Esse tipo de análise permite introduzir, entre os profissionais, a racionalidade econômica, não com o intuito de substituir a clínica, mas sim integrá-la. Na prática, por meio dessa combinação, os profissionais podem remanejar os recursos das intervenções custo-inefetivas para as custo-efetivas (9).

Em um estudo desenvolvido por Guedes e cols. (2008), foi analisada a relação custo-efetividade dos análogos das prostaglandinas para o tratamento do glaucoma e da hipertensão ocular. $\mathrm{O}$ custo mensal para ser obtida uma redução de $1 \%$ na pressão intraocular foi de $\mathrm{R} \$ 2,22$ para a latanoprosta, $\mathrm{R} \$ 1,53$ para a travoprosta, $\mathrm{R} \$ 1,50$ para a bimatoprosta de $3 \mathrm{~mL}$ e $\mathrm{R} \$ 1,35$ para a bimatoprosta de $5 \mathrm{~mL}$. Nesse caso, a bimatoprosta de $5 \mathrm{~mL}$ apresenta a melhor relação custo-efetividade, seguida pela bimatoprosta de $3 \mathrm{~mL}$ e a travoprosta. A latanoprosta foi a que apresentou a pior relação custo-efetividade (14).

Custo-utilidade. Mede a quantidade e qualidade de vida empregando o conceito de utilidade, que se refere à satisfação obtida pelo paciente ante o impacto de uma intervenção de saúde. Considerando o tipo mais complexo de análise, é, objetivamente, a análise custo-efetividade acrescida do ponto de vista do paciente. Os resultados são expressos como o quociente custo/AVAQ (Anos de Vida Ajustados por Qualidade) $(2,4)$. A AVAQ é uma medida de utilidade que relaciona quantidade (variação na mortalidade) e qualidade de vida (variação na morbidade) de um paciente, cuja medição é feita por intermédio de uma escala que possui dois pontos extremos: 0 (morte) e 1 (saúde perfeita), podendo haver também valores negativos que correspondem a estados de saúde considerados, pelo paciente, piores que a própria morte $(4,10)$. Por exemplo, um ano de vida com hemiparesia pode ser equivalente a 0,5 anos de vida com perfeita saúde, ou 0,5 AVAQ (2).

A esperança de vida é medida em anos; a qualidade de vida relacionada à saúde é aferida por meio da aplicação de instrumentos genéricos e específicos. Os instrumentos genéricos geralmente englobam várias dimensões da QVRS (por exemplo, função física, psicológica, social, cognitiva, bem-estar geral, status econômico) e podem ser aplicados à população de pacientes em geral, ou a grupos específicos (2).
Os resultados provenientes são, ainda, submetidos à análise do tipo incremental e de sensibilidade, no sentido de confrontar os números e validar as conclusões do estudo (2).

A análise incremental permite relacionar os custos e resultados de cada alternativa investigada para comparação da eficiência. Para realizá-la, divide-se a diferença dos custos das opções pela diferença dos resultados das mesmas (2). Ela compara os custos adicionais de uma estratégia em relação à outra, comparando com a proporção adicional de efeitos, benefícios ou utilidades que oferecem (15).

A análise de sensibilidade visa testar até que ponto as oscilações nas variáveis relevantes do estudo podem afetar as con clusões. Este tipo de análise parte do pressuposto que, na prática, nem sempre é possível conhecer todos os valores (monetários, percentuais) necessários para realizar uma avaliação farmacoeconômica, pois ocorre um certo grau de incerteza nas suposições e estimativas feitas pelo pesquisador (2).

A análise de custo-utilidade deve ser empregada sempre que a qualidade de vida é importante (como dor, desconforto), incorporando dados de morbidade nas avaliações. É considerado o estudo mais difícil e trabalhoso de ser realizado (7).

É aplicável em estudos voltados para a comparação dos diferentes tratamentos direcionados principalmente a pacientes crônicos (1). Por exemplo, se dois produtos farmacêuticos têm diferentes desfechos com base no número de anos de vida poupados, mas acredita-se que a qualidade de cada ano de vida para os pacientes que estão recebendo um dos dois tratamentos seja muito similar, os ajustes de qualidade podem não ser necessários. Entretanto, em muitos casos (por exemplo, no tratamento do câncer), tanto a sobrevida quanto a qualidade de vida são diferentes, dependendo da terapia selecionada. Às vezes, os tratamentos que mais aumentam a sobrevida são também os mais tóxicos, sendo assim, nesses casos, necessária uma medida que incorpore tanto a sobrevida quanto a qualidade de vida (5).

A principal vantagem dos estudos de custo-utilidade é que os mesmos permitem a comparação entre dife- 
rentes estratégias de intervenção em saúde direcionadas a diferentes condições de saúde. Como exemplo, avaliações de custo-utilidade de uma estratégia de tratamento de câncer de cólon podem ser comparadas com uma estratégia de tratamento de dislipidemia, considerando o benefício em saúde na população como AVAQ (12).

Um exemplo de análise custo-utilidade foi um estudo realizado com pacientes com hérnia de disco, onde foi comparado o tratamento cirúrgico com a terapia conservadora. Por meio de questionários aplicados durante dois anos de seguimento, pode ser estimado o AVAQ. Foram considerados os custos diretos (diagnóstico, tratamento, internação e reabilitação) e os indiretos (diminuição da produtividade e perda social) (9).

Os resultados evidenciaram que em 1,5 ano de seguimento houve um ganho de 0,363 AVAQ à cirurgia, enquanto que o grupo que realizou a terapia conservadora teve um ganho de 0,36 AVAQ a um custo de US\$
44.638. O custo direto representou $24 \%$ do custo total do primeiro grupo e apenas $5 \%$ do segundo. Com isso, concluiu-se que o custo foi de US\$4.648/AVAQ, indicando que a intervenção cirúrgica para o tratamento da hérnia de disco lombar foi melhor, por oferecer melhor desfecho com custo adicional satisfatório (9).

$\mathrm{Na}$ pesquisa desenvolvida por Lindner e colaboradores (2009) foi avaliada a relação custo-utilidade de medicamentos antipsicóticos de primeira e segunda geração para o tratamento da esquizofrenia. Foram analisados os custos do tratamento com haloperidol, risperidona e olanzapina. Os antipsicóticos de segunda geração são disponibilizados pelo Sistema Único de Saúde (SUS) no tratamento da esquizofrenia, quando os de primeira geração não apresentam efetividade pois apresentam custo elevado quando comparado aos de primeira geração (16). Na Tabela 4 estão expostos os resultados de custo-utilidades após período de 5 anos de uso dos antipsicóticos.

Tabela 4. Custo-utilidade da utilização de diferentes antipsicóticos após período de 5 anos (16).

\begin{tabular}{|c|c|c|c|c|c|c|}
\hline Antipsicótico & $\begin{array}{c}\text { Utilidade } \\
\text { (AVAQ) }\end{array}$ & $\begin{array}{c}\text { Utilidade } \\
\text { incremental } \\
\text { (AVAQ) }\end{array}$ & $\begin{array}{c}\text { Custo Total } \\
\text { (US\$) }\end{array}$ & $\begin{array}{c}\text { Custo } \\
\text { incremental } \\
\text { (US\$) }\end{array}$ & $\begin{array}{c}\text { Razão de custo- } \\
\text { utilidade (US\$/ } \\
\text { AVAQ) }\end{array}$ & $\begin{array}{c}\text { Custo-utilidade } \\
\text { incremental } \\
\text { (US\$) }\end{array}$ \\
\hline Haloperidol & 4,1647 & & 3935,15 & & 944,89 & \\
\hline Risperidona & 4,2156 & 0,0509 & 5964,57 & 2029,43 & 1414,90 & 39890,33 \\
\hline Olanzapina & 4,2189 & 0,0034 & 10423,12 & 4458,54 & 2470,57 & $1.329 .394,88$ \\
\hline
\end{tabular}

$A V A Q=$ Anos de Vida Ajustados por Qualidade

Como pode ser observado, para quem inicia o tratamento com haloperidol, ao final de cinco anos, a estimativa é de 4,1647 AVAQ ao custo de US $\$ 3935,15$. A utilidade da risperidona e da olanzapina foram praticamente idênticas; entretanto o custo total de tratamento com a olanzapina foi quase o dobro do valor. De acordo com estes resultados, a opção de início de tratamento com a olanzapina se torna desfavorável, visto que seu custo incremental, ou seja, o valor monetário para se alcançar uma unidade adicional de AVAQ, se mostra muito elevado em relação às demais terapias medicamentosas.

Com relação às medidas de utilidade, os antipsicóticos de segunda geração apresentaram os melhores resultados quando comparados ao haloperidol, devido à maior probabilidade deste em causar efeitos adversos, recaídas e internações hospitala- res, que diminuem a qualidade de vida do paciente, tornando a risperidona a melhor alternativa de tratamento quando o haloperidol não apresentar os efeitos desejáveis.

\section{CONCLUSÃO}

A farmacoeconomia ainda é uma área recente no Brasil, porém tem se mostrado essencial para as tomadas de decisões quanto aos recursos farmacológicos, proporcionando ganhos para o Sistema Único de Saúde, tanto em termos financeiros quanto terapêuticos, por meio da escolha da melhor opção tanto para o paciente quanto para a instituição hospitalar.

A implementação dos estudos farmacoeconômicos é uma importante estratégia para a racionalização terapêutica, permitindo avaliar diferentes variáveis, como o 
custo, a eficácia, o benefício, a utilidade e a eficiência de diferentes tratamentos.

Torna-se, assim, de suma importância a capacitação de profissionais na área hospitalar e a formação de equipes multidisciplinares para o desenvolvimento dos estudos farmacoeconômicos, permitindo a escolha da análise mais adequada para certo tratamento e o cálculo de maneira fidedigna de todos os custos hospitalares para determinada doença, conciliando as necessidades terapêuticas com as possibilidades de custeio do hospital.

\section{REFERÊNCIAS}

1. Alves NDC, Santos TC dos, Rodrigues CR, Castro HC, 1. Areda CA; Bonizio RC; Freitas O. Pharmacoeconomy: an indispensable tool for the rationalization of health costs. Braz J Pharm Sci 2011; 47 (2): 231-240.

2. Secoli SR; Padilha KG; Litvoc J; Maeda ST. Farmacoeconomia: perspectiva emergente no processo de tomada de decisão. Cien Saude Colet 2005; 10 : 287-296.

3. Teich V. Farmacoeconomia: uso de avaliações econômicas para decisão sobre a incorporação de novas tecnologias ao Sistema de Saúde Brasileiro. Astra Zeneca 2011 Mai [acessado em 2014 abr 10] 8p.

4. Secoli SR; Nita ME; Ono-nita SK; Nobre M. Avaliação de tecnologia em saúde: a análise de custo-efetividade. Arq Gastroenterol 2010; 47(4): 329-333.

5. Rascati KL. Introdução à farmacoeconomia. Porto Alegre: Artmed; 2010, 280p.

6. Pereira RLP; Areda CA; Greco KV. A importância da farmacoeconomia na gestão da saúde hospitalar. Rev Racine 2007; 17 (101): 98-100.

7. Guimarães HP; Barbosa LM; Laranjeira LN; Avezum A. Estudos de farmacoeconomia e análises econômicas: conceitos básicos. Rev Bras Hipertens 2007; 14 (4): 265268.

8. Sena OS; Silva DMC; Brito AMG; Rios MC. Farmacoeconomia: Análise dos custos das prescrições medicamentosas na unidade de terapia intensiva de um hospital em Aracuju/SE. Infarma 2010; 22 (9/10): 17-21.

9. Tonon LM; Tomo TT; Secoli SR. Farmacoeconomia: aná- lise de uma perspectiva inovadora na prática clínica da enfermaria. Texto Contexto Enferm 2008; 17 (1): 177-182.

10. Mota DM. Avaliação farmacoeconômica: instrumentos de medida dos benefícios na atenção farmacêutica. Acta Farm. Bonaerense 2003; 22 (1): 73-80.

11. Rodriguez C. Farmacoeconomía aplicada a la antibióticoterapia. Acta Farm. Bonaer. 2004; 23 (2): 226-230.

12. BRASIL. Avaliação econômica em saúde: desafios para gestão no Sistema Único de Saúde. Ministério da Saúde 2008, 104p.

13. Zahdi MR; Maluf I; Maluf EMCP. Hepatite A: avaliação do custo-benefício da prevenção pela vacina - Paraná, Brasil. Rev Bras Med Fam e Com 2009; 16 (4): 281-288.

14. Guedes RAP; Guedes VMP; Chaoubah A. Custo-efetividade dos análogos das prostaglandinas no Brasil. Rev Bras Oftalmol 2008; 67 (6): 281-286.

15. Brandão CMR; Machado GPM; Acurcio FA. Análise farmacoeconômica das estratégias de tratamento da osteoporose em mulheres pós-menopausa: uma revisão sistemática. Rev Bras Reumatol 2012; 52 (6): 912-937.

16. Lindner LM; Marasciulo AC; Farias MR; Grohs GEM. Avaliação econômica do tratamento da esquizofrenia com antipsicóticos no Sistema Único de Saúde. Rev Saúde Pública 2009; 43 (1): 62-69.

17. Herrera MMC; Quiñones WH. Aplicación de la farmacoeconomía a los resultados de la medicación para la curación de las úlceras pépticas. Rev Cubana Farm 2000; 34 (3): 175-180. 